\title{
What do we really know about benign prostatic hyperplasia and lower urinary tract symptoms in adult men?
}

\author{
Matthias Oelke • Martin C. Michel
}

Received: 8 February 2011 / Accepted: 15 February 2011 / Published online: 26 February 2011

(C) The Author(s) 2011. This article is published with open access at Springerlink.com

The guest editors of this topic issue on basic science, epidemiology and assessment of lower urinary tract symptoms (LUTS) in adult men with or without benign prostatic hyperplasia $(\mathrm{BPH})$ have invited leading urologists to contribute articles to one of the above-mentioned topics. Additionally, several original scientific articles on the same topics have been added to this issue to sharpen the reader's view and emphasize the complexity of this medical problem that is responsible for healthy seeking behaviour of an increasingly large number of men in ageing societies.

Our urology teachers lived in an easily understandable world. When dealing with LUTS in adult men, it was postulated that LUTS always originated from bladder outlet obstruction (BOO) due to benign prostatic enlargement (BPE). It was assumed that there was a linear relationship between BPE, BOO, and LUTS. Hence, it was logical to wait with treatment when the patients had only mild symptoms or BPE because BOO could not be severe in these cases. In men with large prostates or severe or treatment resistant LUTS, surgical procedures, usually performed as open removal or later as transurethral resection of the prostatic adenoma, needed to be applied to get rid of $\mathrm{BOO}$ and secondary of LUTS.

In 2011, this ancient vision on LUTS has proven to be untrue. Our understanding of LUTS and BPH has changed dramatically when it became clear that the linear relation-

\section{Oelke ( $\square)$}

Department of Urology, Hannover Medical School, Carl-Neuberg-Str. 1, 30625 Hannover, Germany

e-mail: oelke.matthias@mh-hannover.de

\section{C. Michel}

Department of Pharmacology and Pharmacotherapy, Academic Medical Centre, University of Amsterdam, Amsterdam, The Netherlands ship between BPE-BOO-LUTS is untrue for the majority of men. It also became obvious that histological BPH (or related conditions) is not the only origin of LUTS but is rather multifactorial as, for example, detrusor overactivity, detrusor underactivity, or nocturnal polyuria may also cause similar symptoms. Therefore, assessment and differentiation between the various causes of LUTS have become more challenging during the last decades. Various new drugs and surgical treatments contributed to this complexity. If urologists do not want to use the "trial and error" approach for their patients, they should understand and distinguish the various causes of LUTS and learn how to apply the appropriate tests to prove and treat either condition.

Let us shortly reflect what we really know about BPH or male LUTS. Do we know

- the underlying cause(s) of BPH, detrusor overactivity, detrusor underactivity, or nocturnal polyuria in our patients?

- if community-dwelling men are similar to patients we see in our offices?

- what the exact reasons are why patients seek medical help?

- why some men develop serious medical problems (e.g. upper urinary tract dilatation and renal insufficiency) while most others do not?

- whom we should primarily treat with drugs and whom with surgery?

- what drugs or combinations thereof we should use in men with particular symptoms or conditions?

- what surgery we have to use for which patient?

We should be honest enough to admit that much of our assessment and treatment strategy is gut feeling and "trial and error". Despite learning a lot about the complexity of 
LUTS in the last three or four decades, this does not necessarily mean that we really understand the individual patient in our office. This special issue aims to add new mosaic stones to the general picture, but it would be precocious to assume that we solve the entire problem within the near future.

\section{Conflict of interest None.}

Open Access This article is distributed under the terms of the Creative Commons Attribution Noncommercial License which permits any noncommercial use, distribution, and reproduction in any medium, provided the original author(s) and source are credited. 